We observed a slightly itchy, tumid, violaceous plaque in our patient only in the left retroauricular region and classic lichen planus lesions or systemic symptoms of hypothyroidism were not present. Histopathological examination, besides the pathognomonic findings of lichen planus, revealed band-like lichenoid infiltrate surrounding the follicles and cysts. Therefore, identifying this entity was critical to reach the LPFT diagnosis and to detect the underlying autoimmune disease.

LPFT tends to be chronic, relapsing, and hard to treat. Consequently, all current treatments available are generally disappointing. In our patient, as LPFT is hard to cure and was localized in a small area, clobetasol propionate cream $0.05 \%$ therapy is recommended despite the retroauricular localization of the lesion. Additionally, we warned our patient to come to our endocrinology department for control as soon as she noticed symptoms of hypothyroidism or every 6-12 months.

We decided to report on this case not only because LPFT is a very rare clinical variant, but also because it was observed for the first time with autoimmune thyroiditis in the present case. $\square$

\section{REFERENCES}

1. Belaïch S, Bonvalet D, Civatte J. Follicular tumidus retro-auricular lichen planus (authors transI). Ann Dermatol Venereol. 1977;104:147-50

2. Grupper Ch, Bensoussan L, Beltzer-Garelly E. Lichen plan folliculaire tumidus rétro-auriculaire bilateral associé a un lichen plan buccal banal leucokératosique et erosif. Ann Dermatol Venereol 1977;104:151.

3. Baptista AP, Rodrigues JB, Cortesão J. Follicular lichen planus tumidus. Ann Dermatol Venereol. 1980;107:1057-9.

4. Jiménez-Gallo D, Albarrán-Planelles C, Linares-Barrios M, Martínez-Rodríguez A, Báez-Perea JM, González-Fernández JA. Facial follicular cysts: a case of lichen planus follicularis tumidus? J Cutan Pathol. 2013;40:818-22.

5. Assouly P, Reygagne P. Lichen planopilaris: update on diagnosis and treatment. Semin Cutan Med Surg. 2009;28:3-10.

MAILING ADDRESS

Hatice Kaya Ozden

Kocaeli Derince Training and Research Hospital

Dermatology Department

41900 Kocaeli / Turkey

E-mail:hatcek@gmail.com

How to cite this article: Ozden HK, Polat M, Astarci MH. Coexistence of lichen planus follicularis tumidus and Hashimoto's thyroiditis. An Bras Dermatol. 2017;92(4):585-7.

\title{
Symmetrical drug-related intertriginous and flexural exanthema induced by clarithromycin*
}

\author{
Catarina Moreira ${ }^{1,2}$ \\ Ana Paula Cunha ${ }^{1}$
}

\author{
Maria João Cruz ${ }^{1,2}$ \\ Filomena Azevedo
}

DOI: http:/ / dx.doi.org/10.1590/abd1806-4841.20176125

Dear Editor,

A 43-year-old man with no relevant personal background or chronic medication presented with a symmetrical and sharply demarcated erythema on the buttocks and both inner thighs, with a V-shaped pattern, also affecting the lower abdomen and cubital and axillary folds (Figure 1). The patient denied other symptoms or fever. Blood cell count and serum biochemistry analysis were normal. He had been started on clarithromycin for a respiratory infection 7 days before, and denied previous contact with clarithromycin or intake of other medications during the previous weeks. We suspected of cutaneous adverse reaction to clarithromycin, more precisely baboon syndrome, taking into account the pattern of distribution. We suspended the drug and prescribed topical corticosteroids and an antihistamine with significant improvement of the lesions. Two months after complete resolution, we performed patch tests with the Portuguese Contact Dermatitis Group Standard Series and clarithromycin $(1 \%, 5 \%$, and $10 \%$ petrolatum) with a positive result for the drug.

The term "Baboon Syndrome" (BS) was originally introduced to describe a skin eruption resembling the red gluteal area of baboons, noted in 3 patients previously sensitized to mercury, nickel, and ampicillin by topical contact and subsequent systemic exposure to the same substances. ${ }^{1}$

The acronym SDRIFE (Symmetrical Drug-Related Intertriginous and Flexural Exanthema) was proposed for systemically induced cases with the classical baboon distribution pattern and without previous cutaneous sensitization. ${ }^{2}$ In an attempt to better define the terms and classify the cases previously described in the literature, Miyahara et al. divided BS into classical BS (contact allergen-induced, excluding drugs), topical drug-induced BS (contact allergenic drug-induced related to systemic absorption due to topical exposure), systemic drug-induced BS (contact allergenic drug-induced related systemic exposure), and SDRIFE (exposure to

Received on 05.06.2016

Approved by the Advisory Board and accepted for publication on 18.01.2017

* Work performed at the Department of Dermatology and Venereology, Centro Hospitalar de São João EPE - Porto, Portugal.

Financial support: None.

Conflict of interest: None.

${ }^{1}$ Department of Dermatology and Venereology, Centro Hospitalar de São João EPE - Porto, Portugal.

2 Faculty of Medicine, Universidade do Porto - Porto, Portugal.

(C2017 by Anais Brasileiros de Dermatologia 

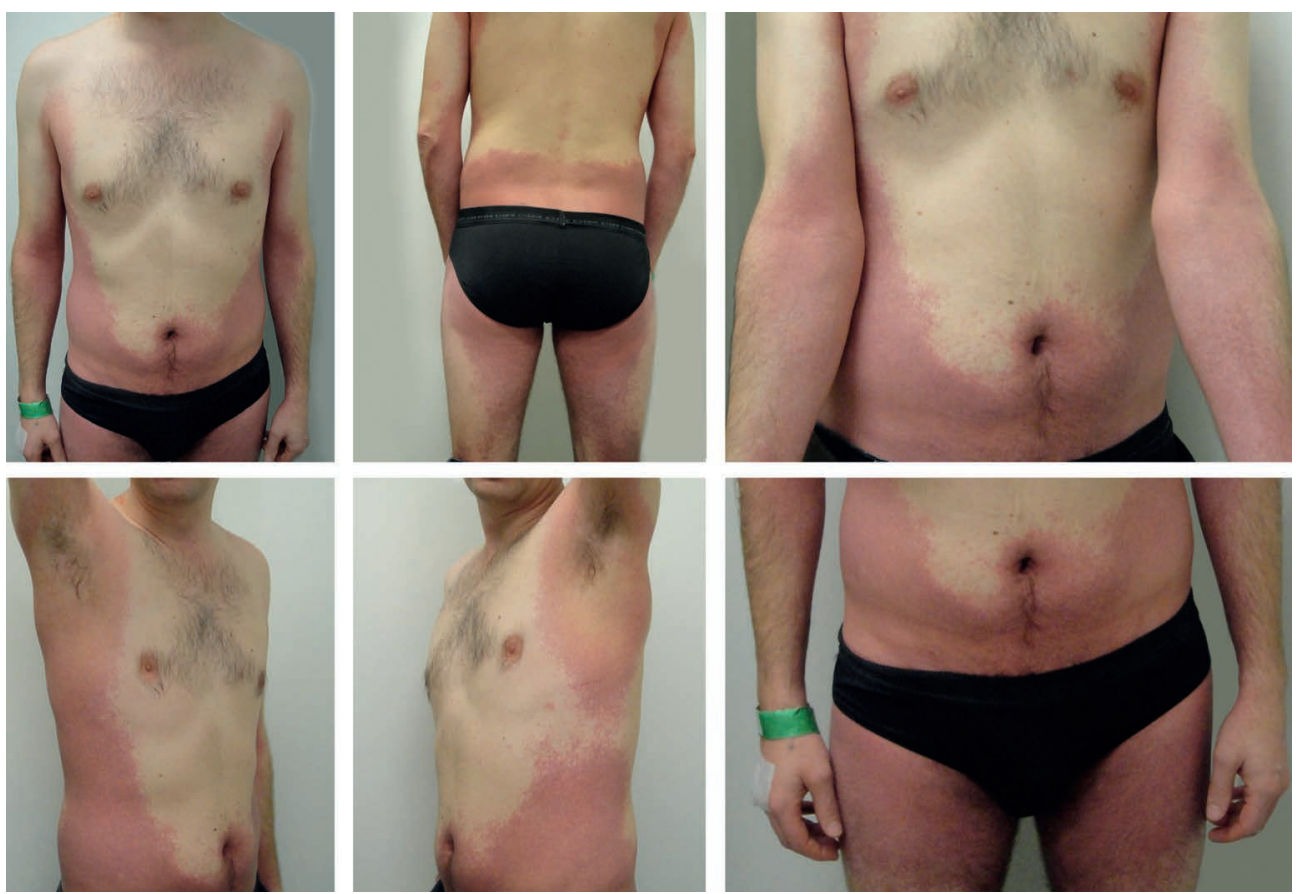

Figure 1:

Clinical presentation. Symmetrical and sharply demarcated erythema on the buttocks and both inner thighs, with a V-shaped pattern, also affecting the lower abdomen and cubital and axillary folds

a systemically administered drug, excluding contact allergens). ${ }^{3}$ In drug-induced cases, the most common agents are $\beta$-lactam antibiotics, especially amoxicillin., ${ }^{2,3}$

BS is characterized by diffuse erythema of the buttocks and anogenital area, usually demarcated by sharp well-defined borders, showing a bathing trunk distribution. Other areas like axillary folds, neck, face, and lips are occasionally involved. Most cases are not accompanied by systemic symptoms. ${ }^{3}$

Although the precise mechanism for its development remains unknown, there is strong evidence for the role of a T-cell mediated delayed-type hypersensitivity reaction. ${ }^{4}$

The diagnosis is generally based on patient history and clinical findings. Laboratory tests and skin biopsy are neither specific nor diagnostic. Although patch tests, lymphocyte transformation tests, and drug provocation tests (DPTs) can be used for diagnosis, DPTs remain the gold standard for determining the offending drug. ${ }^{5}$

Cutaneous lesions resolve spontaneously after the offending drug is discontinued, so treatment is directed toward symptomatic relief. Topical or systemic glucocorticoids may hasten recovery. ${ }^{4}$

We report here a case of clarithromycin-induced BS without a known prior sensitization (clarithromycin-induced SDRIFE). To the best of our knowledge, this is the first case of SDRIFE related to clarithromycin.

This entity should be considered in a patient with a symmetric intertriginous eruption involving multiple body folds in order to avoid a misdiagnosis. A detailed history and review of exposures is essential to guide the search for the etiologic agent. $\square$

\section{REFERENCES}

1. Andersen KE, Hjorth N, Menné T. The baboon syndrome: systemically-induced allergic contact dermatitis. Contact Dermatitis. 1984:10:97-100.

2. Häusermann P, Harr T, Bircher AJ. Baboon syndrome resulting from systemic drugs: is there strife between SDRIFE and allergic contact dermatitis syndrome? Contact Dermatitis. 2004;51:297-310.

3. Miyahara A, Kawashima H, Okubo Y, Hoshika A. A new proposal for a clinicaloriented subclassification of baboon syndrome and a review of baboon syndrome. Asian Pac J Allergy Immunol. 2011;29:150-60.

4. Elmariah SB, Cheung W, Wang N, Kamino H, Pomeranz MK. Systemic drug-related intertriginous and flexural exanthema (SDRIFE). Dermatol Online J. 2009;15:3.

5. Bulur I, Keseroglu HO, Saracoglu ZN, Gönül M. Symmetrical drug-related intertriginous and flexural exanthema (Baboon syndrome) associated with infliximab. J Dermatol Case Rep. 2015;9:12-4.

MAILING ADDRESS:

Ana Catarina da Cunha Moreira

Departmento de Dermatologia e Venereologia,

Centro Hospitalar de São João EPE

Alameda Prof. Hernani Monteiro,

4200-319 - Porto, Portugal

E-mail: cat_moreira@icloud.com

How to cite this article: Moreira C, Cruz MJ, Cunha AP, Azevedo F. Symmetrical drug-related intertriginous and flexural exanthema induced by clarithromycin. An Bras Dermatol. 2017;92(4):587-8. 